\title{
Determination of Thermal Parameters of Concrete by Active Thermographic Measurements
}

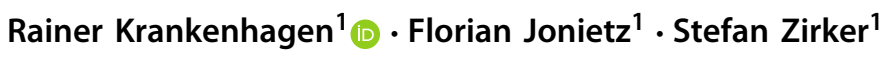

Received: 6 August 2021 / Accepted: 20 February 2022 / Published online: 6 March 2022

(c) The Author(s) 2022

\begin{abstract}
The knowledge of the thermal parameters of a particular concrete is essential for thermal design of a building, but also could help to identify and assess the state of a concrete structure. Active thermography has the potential to be applied onsite and to provide a fast investigation of thermal properties. In this work, three different concrete samples were investigated by active thermography in reflection and in transmission setup. It was found that this method yields the same results without direct contact as the Transient Plane Source (TPS) method as an established inspection tool.
\end{abstract}

Keywords Concrete · Effusivity · Thermal diffusivity · Photothermal · Thermography · Hot disc method · Parker method . Onsite inspection

\section{Introduction}

Although many new materials are being developed and introduced for the construction of buildings, as they offer great potentials for saving energy by e.g., reduced thermal conductivity, concrete is still one of the most widely used materials in the construction industry. However, many kinds of concrete exist and must be chosen for the respective purpose. Not only the mechanical properties are decisive, but also the thermal parameters like heat capacity and thermal conductivity. Often, these are not listed in sufficient detail for the used concrete, as the material mixtures might vary gradually. When examining the concrete in old buildings, the used concrete might not be documented, and its properties must then be determined on-site or by taking samples. A series of recent studies and publications was focused on the determination of the thermal properties of an entire wall as part of an existing building to evaluate the heat energy consumption [1-3]. The reported methods are regarding the entire thickness of the inspected wall in its actual state with moisture and degradation of materials, but last for several hours or even days due to the large thermal inertia. In contrast, the authors of this contribution want to establish a fast method to identify the kind of

Rainer Krankenhagen

rainer.krankenhagen@bam.de

1 Bundesanstalt für Materialforschung und -prüfung, Berlin, Germany concrete onsite by means of thermal properties at the surface. In certain cases those values are needed to enable numerical simulations of a building or component [1]. In a recently developed method for a nondestructive thickness measurement of surface protecting coatings on concrete, the thermal properties of the covered concrete are also required [4]. In contrast, Wang et.al. determined coating thicknesses without knowledge about the actual value of the thermal diffusivity by using a calibration data set for an artificial intelligence approach [5]. Since porosity strongly influence thermal properties [6] some recent research work was focused on the consideration of heat propagation by means of transit times of virtual waves [7]. Here, transit times at known sample thicknesses are exploited to determine a wave velocity strongly correlated with thermal diffusivity and eventually with porosity.

Several methods exist to determine the thermal properties of materials and in particular also of concrete $[6,8]$. One example is the hot disc method or Transient Plane Source (TPS) method, which will serve as a reference method for our measurements [9-12]. Other methods described in the literature include photothermal deflection technique [8], laser spot heating combined with pyrometer point measurements [13], photopyroelectric calorimetry (PPE) [6], time-modulated laser spot heating combined with lock-in thermography [6] or a hotwire technique [14]. The flash method is well established for the measurement of the thermal diffusivity [15] and is even described in related standards [16, 17]. Liu et al. 
applied this method to investigate the influence of moisture in different lightweight mortars on their thermal conductivities [18]. Santhosh et al. used it also to determine the porosity in ceramics [19]. However, most of these methods are laboratory measurements, for which a sample with strong restrictions in size and dimension must be taken.

Active thermography is generally considered as a rather inexpensive and fast method with high flexibility concerning the sample size, which can in principle be conducted onsite on buildings $[1,3,20]$. Therefore, this work examines the feasibility of active thermography for deducing the thermal parameters of concrete, but in a short-term mode. The accuracy of the method will be demonstrated as well as possible drawbacks and difficulties that must be considered when using this method on concrete. Also, active thermography was used to determine the thermal parameters of construction materials in [6], but the method applied there uses timemodulated heating and is far more difficult to realize than the method presented here. Thus, it falls more into the category of laboratory measurements than possible on-site measurements. Furthermore, like the TPS method [9-12], it is based on measuring the local thermal parameters at one point or small region of the sample surface. To measure useful values describing the thermal parameters of concrete as a material mixture, an averaging over larger areas or over a multitude of different single points is required. The method described in this work provides the inspection of a representative area within a minute. Thus, it allows the nondestructive testing of larger structures at selected regions.

In order to demonstrate the performance and validity of this method, three different kinds of concrete were investigated. Finally, the reported results provide the good agreement between TPS method and active thermography with laser pulses not reported so far. The possibility to perform active thermography in reflection mode on concrete offers a way for a real onsite measurement.

This study is organized in different sections:

- Concrete samples: Describes the sample preparation

- TPS measurements

- Measurements in transmission geometry: Method is applied to a separated sample of certain thickness similar to the flash method [21]

- Measurements in reflection geometry: Method is applied to the surface of a sample with unknown thickness

- Discussion: Comparison of the results with values given in literature and standards

\section{Concrete Samples}

Three different types of concrete were selected from the stock: One repair mortar and two lightweight concretes. All samples were cut from a larger block and reveal two different surfaces: the formwork surface and a smoothed plane from the inside dominated by a variety of cut grains. The investigations were focused on the cut side with its smooth surface to enable a better thermal contact for the TPS method.

\subsection{Sample Type 1 (Lightweight Concrete with Red Expanded Clay)}

Figure 1a shows the surface of sample type 1 at the cut side. The thicknesses of the available samples of this type were $1 \mathrm{~cm}$ and $1.5 \mathrm{~cm}$. The length of the square-shaped sample is $10 \mathrm{~cm}$. The photo shows the type of inclusions existent in this sample: Large pores and large black and red colored inclusions. The red inclusions consist of expanded clay. Figure $1 \mathrm{~b}$ shows the same specimen after coating with Mibenco liquid rubber spray leaving the upper right quarter free. This coating is often applied in the field of thermographic testing (discussion see below in Sect. 2.5). Since an additional coating is not favorably for onsite application, one quarter of the sample surface was kept uncoated to enable investigations with and without coatings.

\subsection{Sample Type 2 (Repair Mortar)}

Figure 2 shows the sample type 2. Again, the left photo shows the surface without coating and the photo on the right the same surface after partial coating. The thicknesses of the samples of this type were $1 \mathrm{~cm}$ and $1.5 \mathrm{~cm}$. The diameter of the samples is $10 \mathrm{~cm}$. The photos show the type of inclusions: Larger and smaller pores and small inclusions of differently colored materials. Thus, the composition of this sample is very different from sample type 1 .

\subsection{Sample Type 3 (Lightweight Concrete with Black Expanded Clay)}

Figure 3 shows the sample type 3 . It has a thickness of $1 \mathrm{~cm}$ and a diameter of $10 \mathrm{~cm}$. The surface reveals large pores and inclusions of different size and color. Some of the larger inclusions are black with a porous consistency. 
Fig. 1 a Sample type 1 cut surface. b Sample type 1 cut surface after coating with Mibenco liquid rubber

Fig. 2 a Sample type 2 cut surface. b Sample type 2 cut surface after coating with Mibenco liquid rubber

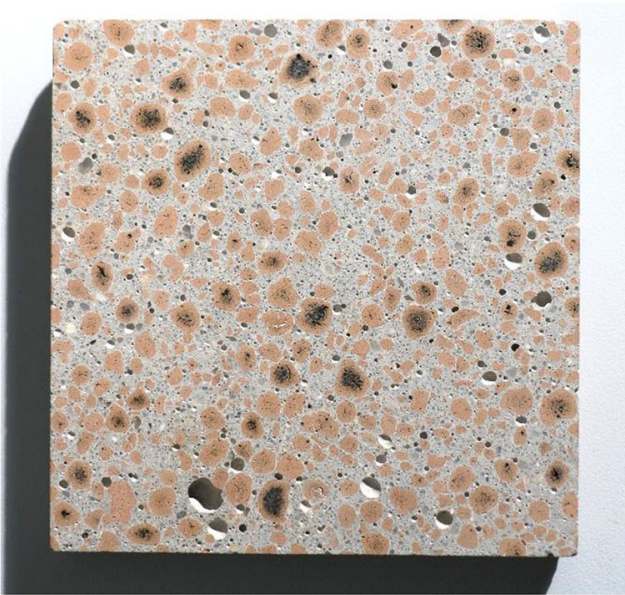

$\mathbf{a}$

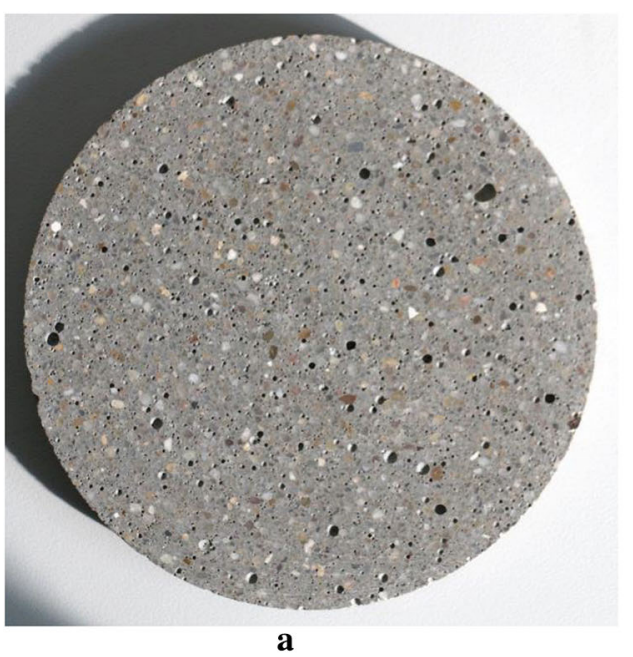

a

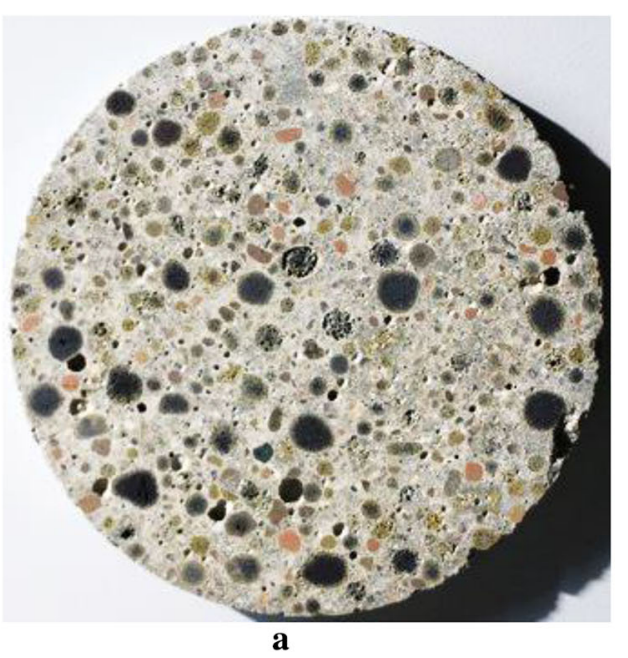

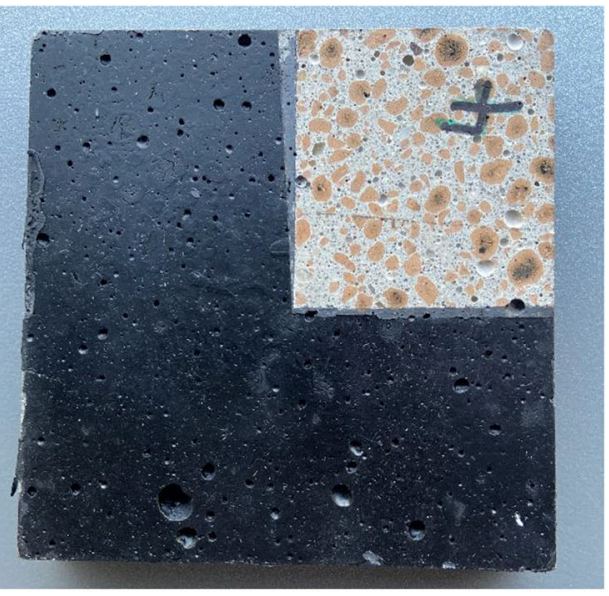

b

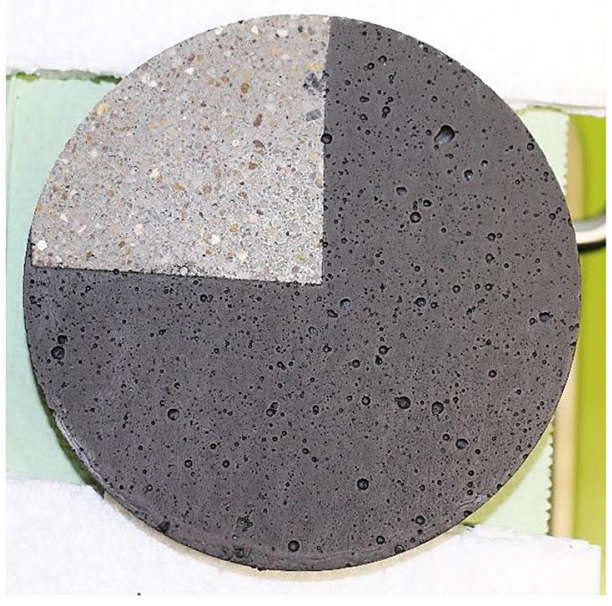

b

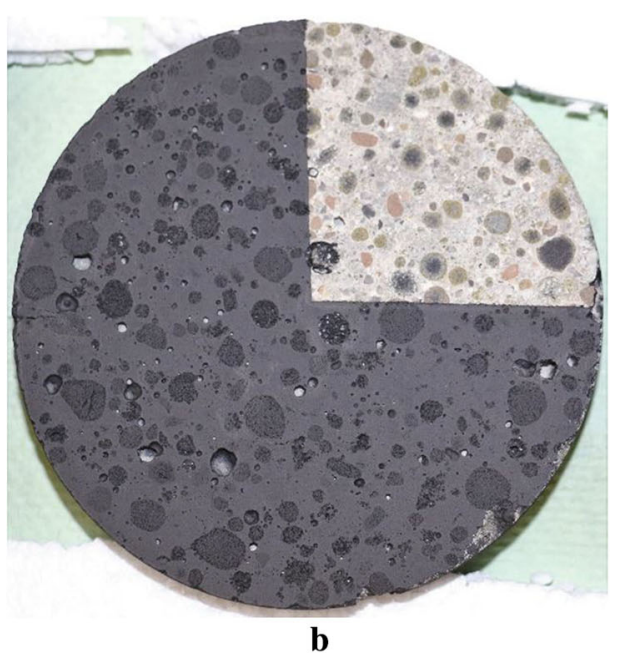

Fig. 3 a Sample type 3 cut surface. b Sample type 3 cut surface after coating with Mibenco liquid rubber 


\subsection{Thermal Conductivities Measured by Transient Plane Source (TPS) Method}

The thermal conductivity measurements were carried out according to the TPS method. In this method, measurements are made with a heated plane sensor element in the shape of a double spiral. The investigations were implemented with the "Hot Disk Thermal Constants Analyser", a measuring system from the company Hot Disk AB. The device TPS 1500 was used [22]. Because of the small sample thicknesses, a probe head with a diameter of only $6.4 \mathrm{~mm}$ had to be used (Kapton sensor 5465). Usually, for porous or inhomogeneous materials, it is recommended to use a sensor with a radius larger than $15 \mathrm{~mm}$ [23]. Since there was only one specimen in each case, measurements were conducted from one side. In a one-sided measurement the sensor is located between the specimen and a material with a known thermal conductivity, which should be significantly lower than that of the specimen (in this case Styrofoam). The setup is demonstrated in Fig. 4.

The measurement accuracy is stated by the manufacturer to be better than $5 \%$ and the reproducibility of the measurements better than $1 \%$ [22].

The small size of the sample, as well as that of the sensor, can have a negative effect on the measurement. Therefore, a series of individual measurements was conducted at the cut side and on coated and uncoated regions of every sample to consider the inhomogeneous composition with larger inclusions. The following Table 1 compiles the results, together with the values given by a German standard for concrete [24], where a curve conductivity vs. density is provided. Thus, the individual measured densities of the investigated samples are also included in Table 1. Density uncertainties were estimated by the give uncertainties of mass dimension measurements. The uncertainties of the conductivity values are the standard deviation as a first approximation because the value distribution was uneven. Only values from the coated regions were regarded in Table 1 because they are expected to be more reliable due to better thermal contact at the surface and larger investigable areas were available. Please note that as a first

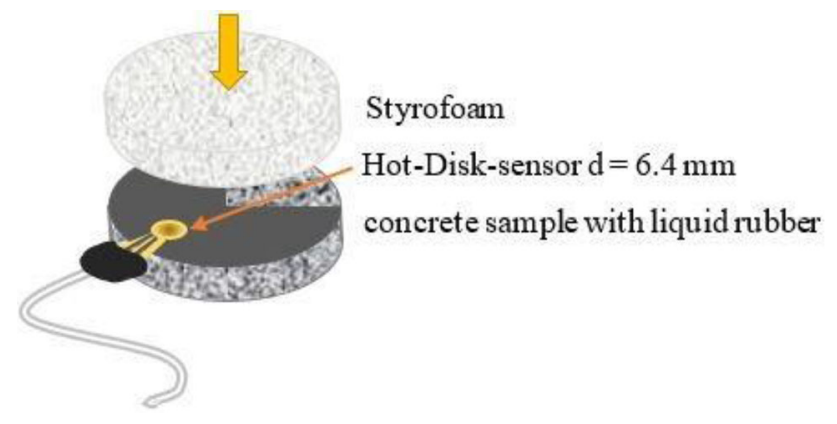

Fig. 4 Setup of the TSP method with the Hot-Disk sensor in one-sided configuration
Table 1 Densities $\rho$, expected and measured values of the conductivity $\mathrm{k}$ of the investigated specimens

\begin{tabular}{llll}
\hline & Sample Type 1 & Sample Type 2 & Sample Type 3 \\
\hline$\rho\left(\mathrm{kg} / \mathrm{m}^{3}\right)$ & $1930 \pm 50$ & $2125 \pm 85$ & $1860 \pm 70$ \\
$\begin{array}{c}\text { number of } \\
\text { measurements }\end{array}$ & 22 & 16 & 7 \\
$\begin{array}{c}k_{\text {measured }}(\mathrm{W} /(\mathrm{m} \\
\mathrm{K}))\end{array}$ & $1.37 \pm 0.03$ & $2.54 \pm 0.05$ & $1.6 \pm 0.20$ \\
$\begin{array}{c}k_{\text {exptected }}(\mathrm{W} /(\mathrm{m} \\
\mathrm{K})) \text { from }[24]\end{array}$ & 1.35 & 1.6 & 1.35 \\
\hline
\end{tabular}

approximation a possible influence of the coating is covered internally by the parameter thermal contact resistance. However, A systematic study of the actual influence of the applied Mibenco coating on the measurement results was not in the focus and was not carried out so far.

The obtained thermal conductivities $k_{\text {measured }}$ comply with the specification of the standard in case of sample types 1 and 3 . On the contrary, sample type 2, the repair mortar, has a significantly higher thermal conductivity than would be expected for normal concrete with this density.

Thus, the investigated kinds of concrete embody a certain bandwidth of material parameters in concrete with medium density. Thus, the following results can be viewed as a general approach to apply active thermography on various types of inhomogeneous construction materials, not limited to a special kind of concrete.

\subsection{Optical Properties of Concrete Surfaces}

Whenever active thermography is conducted in terms of a quantitative data evaluation, two effects have to be regarded: the emissivity of the inspected surface within the sensitivity range of the IR camera and the heating energy absorption actually achieved. Both effects can be handled normally by the application of a black coating, but this is not favorable for many test items. However, some standards even require a black coating to avoid the named effects.

The emissivity of the investigated concrete samples was estimated following the regulations of the standard ASTM E1933 [25] and was found to be very high, that means differences between radiometric measured temperatures and temperatures measured by thermocouple are within the uncertainties. This was found for both: for coated and uncoated surfaces as well. Thus, from an emissivity point of view, a coating is not required in case of the investigated concrete surfaces. Thus, an infrared camera sensitive in the wavelength range between 3 and $5 \mu \mathrm{m}$ with a valid temperature calibration delivers correct temperature values without further corrections. 
Table 2 Obtained absorptivity values of 3 concrete samples and a black rubber coating Mibenco at the laser wavelength of $940 \mathrm{~nm}$

\begin{tabular}{lll}
\hline & Cut side & Formwork side \\
\hline Sample type 1 & $0.625 \pm 0.04$ & $0.585 \pm 0.03$ \\
Sample type 2 & $0.81 \pm 0.01$ & $0.775 \pm 0.02$ \\
Sample type 3 & $0.685 \pm 0.04$ & - \\
Coating Mibenco & $0.95 \pm 0.01$ & - \\
\hline
\end{tabular}

The real energy absorption plays a major role in active thermography because it influences the achieved temperature increase directly and a useful discussion of thermal effects is almost impossible without this number (see [26] and references therein). Since it is known that concrete can have a noticeable reflectance [27] the reflectivity was measured with a spectrometer, the Perkin-Elmer Lambda $1050 \mathrm{~s}$. The wavelength range was limited to the range between 930 and $950 \mathrm{~nm}$, including the wavelength of the laser used as heating source (see Sect. 4). The measurements were conducted five times on every sample on both sides to cover the different components present at the surface. Thus, the specified uncertainties are not determined by the reproducibility of the method but by the different components. And the average values represent an effective value for the entire surface. As to be expected from the visible appearance, sample type 2 is more homogeneous resulting in a much lower spreading of the results.

Actually, the reflectivity $R$ was measured, but it is connected with the absorptivity $A$ by the simple relation $A=1$ $-R$. The obtained absorptivities of the investigated samples are listed in Table 2, which gives the minimum and the maximum value of the measured absorptivities. Spectral curves of various types of concrete are documented in data bases [7] and agree with our data.

The data listed in Table 2 enable the estimation of the real energy density deposited during laser heating of the concrete samples using the known power density of the laser output and the length of the heating pulse, in uncoated as well as in coated parts of the surface.

\section{Thermal Parameters}

The thermal behavior of a material is basically defined by two parameters: Thermal conductivity $k$, which describes the speed, at which heat diffuses through the material, and specific heat capacity $c$, which determines the temperature rise of the material with mass density $\rho$, when a certain amount of heat (i.e., thermal energy) is absorbed. Alternatively, the thermal behavior can be described by a set of two other thermal parameters, which can be deduced from $k, c$ and $\rho$, and vice versa: thermal diffusivity and thermal effusivity.
The speed, at which a temperature rise progresses through the material, is determined by the thermal diffusivity $D$. It is defined as the ratio between the conductivity $k$ and the product of specific heat capacity $c$ and material density $\rho$ :

$D=\frac{k}{\rho c}$

This definition results directly from the heat equation, details are described in textbooks [28].

The thermal effusivity $\varepsilon$ is a measure of the material's ability to exchange thermal energy with its surroundings. For example, when touching two materials at the same temperature (i. e. at room temperature), the material with the higher effusivity feels colder than the material with lower effusivity, because higher effusivity implies that the heat from the touching hand diffuses with higher rate from the contact surface into the bulk of the material, thus, the touching hand will be more cooled at the contact surface. Thus, the temperature at the contact between touching hand and material surface gets lower. The thermal effusivity $\varepsilon$ is defined as:

$\varepsilon=\sqrt{k \rho c}$.

This formulae results from specific solutions of the heat equation, details are also available in textbooks e.g. [29]. Thus, the original thermal properties can also be defined by the two parameters $D$ and $\varepsilon$ :

$k=\varepsilon \cdot \sqrt{D}$

$c=\frac{\varepsilon}{\sqrt{D} \cdot \rho}$

In a simple model with spatially homogeneous Dirac pulse heating of an opaque and isotropic material, where the heat is completely absorbed at the surface, and where all heat losses and a finite sample thickness are completely neglected, the surface temperature-time dependence $T(t)$ can be described by the following equation $[27,29]$ :

$T(t)=\frac{Q}{\sqrt{\pi} \cdot \varepsilon \cdot \sqrt{t}}$

This equation provides a simple relation between the absorbed energy $Q$ and the thermal effusivity $\varepsilon$ of a material when the surface was heated by a short pulse and the temperature transient $T(t)$ is recorded within a certain period after the pulse. Knowing the effusivity allows the estimation of the absorbed energy and vice versa. A successful application of Eq. 5 was reported in [27] demonstrating the power of this simple relationship. 
Fig. 5 Measurement setup in transmission geometry: Sample (upper right quarter remained uncoated) placed between laser heat source and IR-camera. Widened laser beam is indicated

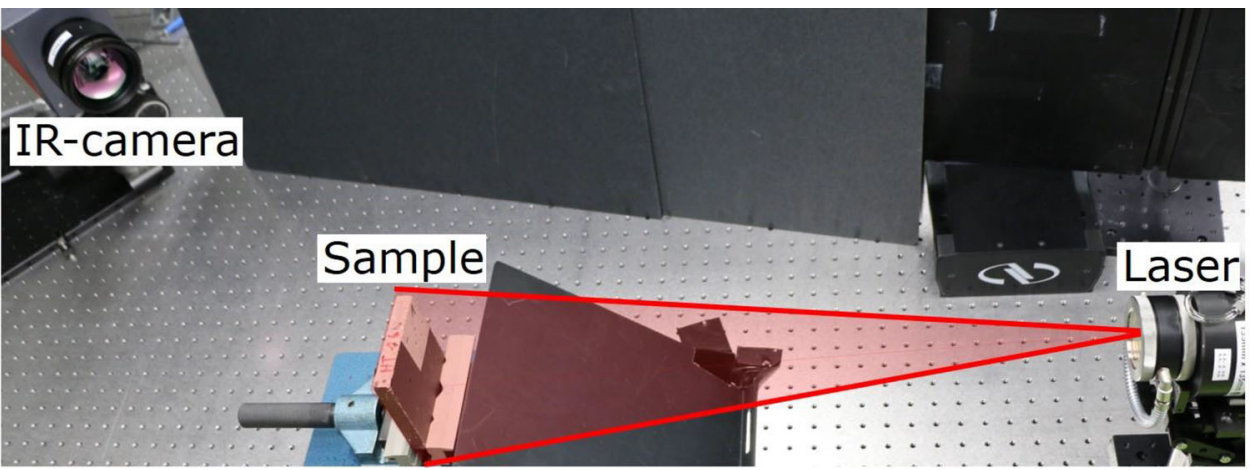

\section{Measurement in Transmission Geometry}

\subsection{Measurement Setup in Transmission Geometry}

In this section, the measurements in transmission geometry are presented. Figure 5 shows the experimental setup. This setup is derived from the Parker-method to determine the thermal diffusivity [21] and is also described in international standards [16, 17, 30-33]. Some standards require a black coating to avoid heat penetration during the heating pulse.

Here are the technical parameters and specifications for the laser system applied as heating source.

Laser system LDM 500 with beam widening optics:

- Output power at the fiber: $540 \mathrm{~W}$

- Wavelength: 935 ... $942 \mathrm{~nm}$ (depending on output power)

- Pulse length: $10 \mathrm{~ms}$ until infinite

- Beam width after optics: square with $135 \times 135 \mathrm{~mm}^{2}$ in $60 \mathrm{~cm}$ distance

- Power density measured: $2.8 \pm 0.2 \mathrm{~W} / \mathrm{cm}^{2}$

Please note the large cross-section area of the widened laser beam covering the complete sample area of $10 \mathrm{~cm}$ diameter, and also shining past the edge. The technical specifications of the IR camera Image IR 8300 with $25 \mathrm{~mm}$ objective are:

- Cooled InSb detector array with $640 \times 512$ pixels

- Frame rate: $300 \mathrm{~Hz}$ full frame

- Sensitivity range: $2.5 \ldots 5.7 \mu \mathrm{m}$

- NETD: $<25 \mathrm{mK}$

- Integration time $\left(10 \ldots 100{ }^{\circ} \mathrm{C}\right): 0.5 \mathrm{~ms}$

The settings for the experiments were:

- Heating pulse length: $5 \mathrm{~s}$

- Observation period: $5 \mathrm{~min}$

- Frame rate: $40 \mathrm{~Hz}$

- Distance between camera and sample surface: $51 \mathrm{~cm}$

- Resulting spatial resolution: 6 pixels/mm
Please note the sensitivity range of the camera is clearly separated from the laser wavelength. Hence, the detected thermal signal of the sample is not disturbed by radiation from the heating source. The sample is placed between the laser optics and the tilted infrared camera in relation to the optical axis. This setup protects the camera objective from direct laser irradiation and allows a direct observation of the heating phase at the sample edge and thus an exact determination of the start time (see Fig. 6a) and the duration of the heating period. A synchronization of camera and laser was therefore not necessary.

\subsection{Results of Measurements in Transmission Geometry}

The selected long heating time of $5 \mathrm{~s}$ was necessary to sufficiently heat up the sample in order to obtain a clearly detectable temperature rise of a few Kelvin at the rear side of the sample. The time at which the heating began was determined, and the thermal images before the irradiation where averaged and subtracted as background, which is in accordance with the procedures described in [21] and in [16, 17, 30-33]. In this way, only temperature differences related to the unheated state will be analyzed. Subsequently, thermal images recorded before the heating period were discarded.

The following figure provides 3 thermograms of a sample type 1 in transmission geometry recorded at different times. Figure $6 \mathrm{a}$ demonstrates the possibility to record the beginning of the heating period due to the tilted orientation of the IR camera, where the heated front side appears as a thin contour line mainly at the left edge of the specimen. Additionally, a line shaped horizontal area occurred at the lower left edge probably due to grazing incidence of the laser light at the bottom. The tilted orientation also enables a cross-section view on the specimen giving a rough impression of the transversal heat profile inside the specimen (included with a black dotted line in Fig. 6b). Figure $6 \mathrm{~b}$ shows the thermogram after the $5 \mathrm{~s}$ long heating period. Using the same temperature scale as in Fig. 6a demonstrates that no temperature increase appeared at the rear side due to the large thickness of the specimen. 


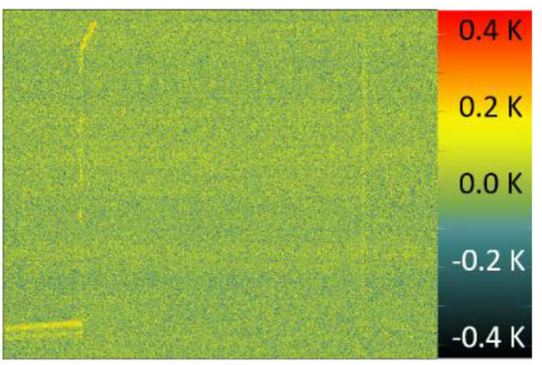

a

Fig. 6 a Difference thermogram of the sample type 1, recorded at the beginning of the heating period, the yellow lines are heated edges of the sample. b difference thermogram of the sample type 1, recorded at the end of the heating period; the black dotted rectangle includes the cross-

However, the front side temperature increased to $15 \mathrm{~K}$ which was strongly outside of the selected temperature range. Thus, the related left contour and the heated bottom areas are displayed as overridden regions. The cross-section view reveals that the detectable heat front inside has reached the half thickness. Figure $6 \mathrm{c}$ displays the recorded temperature distribution $100 \mathrm{~s}$ later when the maximum temperature level was reached. The uncoated quarter can be detected clearly at the upper right corner. Here, the inhomogeneous composition of the concrete can be recognized by a blotchy texture. In contrast, the coating on the other part causes an almost homogeneous appearance. The temperature level is roughly the same as to be expected from the emissivity measurement results (see Sect. 2.5).

Two further effects were observed at Fig. 6c:

(i) Lower temperatures at the lower specimen edge because of heat losses due to heat conduction into the specimen mounting

(ii) A warmer frame within the uncoated region at the interface between coated and uncoated region probably since the same coating pattern is present at the other side leading to an inhomogeneous energy absorption as discussed in Sect. 2.5, thus the coated region was stronger heated causing a heat flow into the uncoated region due to internal heat conduction

Both effects influence the temperature transients in the affected areas and must be taken into account when evaluating the data.

\subsubsection{Parker Method}

Since the international standards [16, 17, 30-33] usually request the sample to be coated by a blackening substance, a region of interest in the coated sample area (left black rectangle in Fig. 6c) was analyzed. Additionally, a region

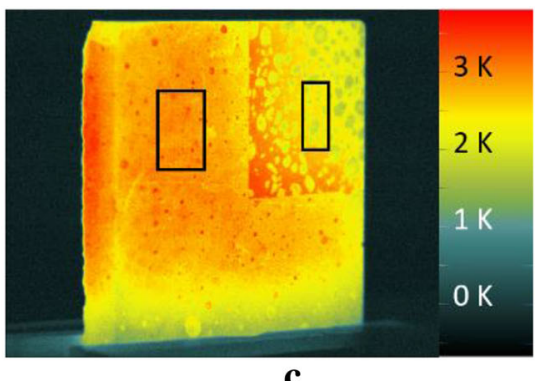

b

section part of the sample due to the tilted orientation. c Difference thermogram of the sample type 1 with maximum temperature $(100 \mathrm{~s}$ after the heating period), the black rectangles indicate the regions of interest, where the transients were extracted

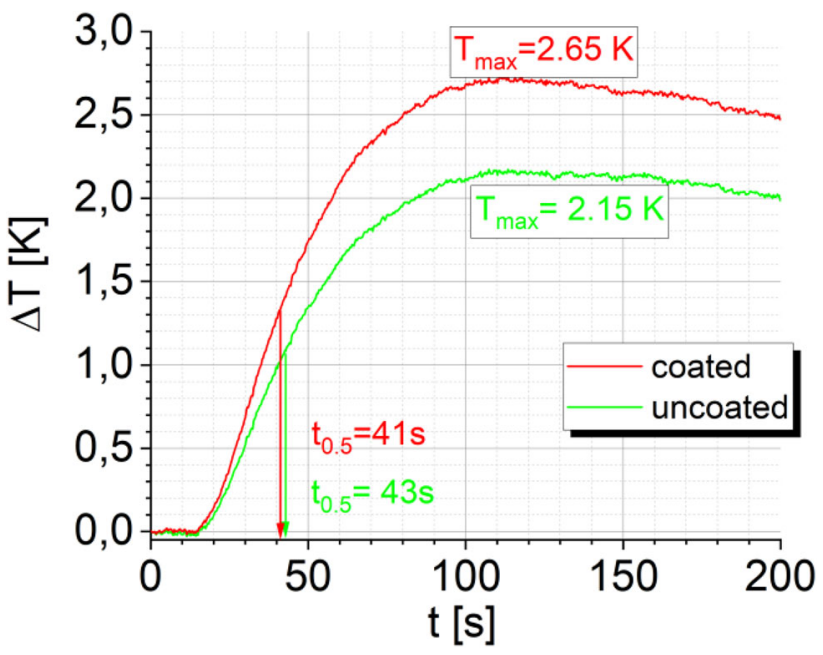

Fig. 7 Temperature transients recorded in transmission setup with $5 \mathrm{~s}$ heating period within the uncoated part (upper right corner) and the coated part (see black rectangles in Fig. 6c), the arrows indicate the related $\mathrm{t}_{0.5}$ values used for the diffusivity calculation

in the uncoated part was also analyzed (right black rectangle in Fig. 6c) to study the influence of the coating in the case of concrete to be investigated. The corresponding transients for the Parker analysis are displayed in Fig. 7. The pulse has started at $1.275 \mathrm{~s}$ on the time scale (with respect to the thermal sequence of the observation) and was $5 \mathrm{~s}$ long. Both transients have a very similar shape, but the coated part reached a higher maximum temperature due to larger energy input at the front side (see statement above). Both transients have a clear maximum followed by a slowly decreasing part. The indicated half maximum times are very similar in both cases. The influence of the finite pulse length instead of a Dirac pulse was considered by shifting the zero point of the time scale to the half of the pulse length. The obtained values for the investigated specimen are listed in Table 3, in comparison to the values of the TPS method and of the quadrupole method. The reported uncertainty range is valid for all sam- 
Table 3 Diffusivities measured with TPS method and with active thermography (all values in $\mathrm{mm}^{2} / \mathrm{s}$ ) for the investigated specimens, the stated uncertainties are representative for all samples, details are given in the related text parts above and below

\begin{tabular}{llll}
\hline & $\begin{array}{l}\text { Sample type 1 } \\
\text { Probe 4 }\end{array}$ & $\begin{array}{l}\text { Sample type 2 } \\
\text { Probe 6 }\end{array}$ & $\begin{array}{l}\text { Sample type 3 } \\
\text { Probe 5 }\end{array}$ \\
\hline $\begin{array}{l}\text { TPS coated } \\
\begin{array}{c}\text { Parker method } \\
\text { (coated and }\end{array}\end{array}$ & $0.68 \pm 0.1$ & $1.36 \pm 0.09$ & $0.81 \pm 0.15$ \\
$\begin{array}{c}\text { uncoated) } \\
\text { Quadrupole } \\
\text { method } \\
\text { coated }\end{array}$ & 0.72 & 1.36 & 0.95 \\
$\begin{array}{c}\text { Quadrupole } \\
\text { method } \\
\text { uncoated }\end{array}$ & $0.71 \pm 0.015$ & 1.32 & 0.81 \\
\hline
\end{tabular}

ples and results from varying the time value by $1 \mathrm{~s}$ in both directions, while the other measured variables $\mathrm{T}$ and thickness $d$ have only a minor influence on the precision of the final result.

In case of sample type 2 both methods agree well but in case of the other both the Parker method yields certainly higher values. Thus, another evaluation method (the Quadrupole method see Sect. 4.2) was carried out to compensate the simplifications of the Parker method: the neglect of a finite pulse length as well as thermal losses.

\subsubsection{Quadrupole Method}

For the work presented here, the data were analyzed and fitted (red line) applying the Quadrupole method [34] with a refined model based on Salazar et al. [35], which eliminates some major simplifications of the previous model. It allows the consideration of a finite heating time, of thermal losses and of semitransparent materials. All heat losses are included in a common heat transfer coefficient $h$. All heat losses mean here: it contains convective losses, radiative losses, and conductive losses to the sample mounting (see Fig. $6 c$ at the lower edge). The initial value for $\mathrm{h}$ was estimated manually focusing only on the decreasing part of the transient. Semitransparency is regarded by an absorption coefficient $\alpha$ to describe a penetration depth of the optical excitation pulse for semitransparent materials. It was set to the maximum value (here $88,000 / \mathrm{m}$ ) because the coating as well as the concrete are assumed to be opaque.

In order to quantify the quality of the fitting results the sum of the squared differences of the logarithms of experimental and model data was used as a quality parameter qp. Thus, smaller values indicate better fits but the value itself has no real physical meaning.
For the fitting routine, the absorption coefficient $\alpha$ was set to a constant maximum value to describe a completely opaque material. The conductivity $\mathrm{k}$ was set to be constant and the energy input $Q$ in $J / m^{2}$ was a free fit parameter. Figure 8a shows the direct comparison of an averaged experimental data set and the fitted transient. The quality of the fit is well, and the obtained diffusivity is certainly smaller as by the Parker method.

An improved fit could be obtained by setting $\alpha$, and also h, as free fit parameters, as can be seen in Fig. $8 \mathrm{~b}$. The quality parameter was reduced by a factor of 3 and the obtained diffusivity value is near to the one measured by the TPS method. The reduced value for $\alpha$ represents a penetration depth of about $1 \mathrm{~mm}$, although the surface was coated. This is either caused by micropores in the coating resulting in a certain penetration depth or it is a specific property of the fit model. The same fit configuration was applied to the transient in the uncoated region and the results were very similar (see Fig. 9).

The reported uncertainty for the obtained diffusivity value in Table 4 was estimated by the variation of the thickness by $\pm 0.1 \mathrm{~mm}$ as the dominant uncertainty source of this method. The thickness was measured with a micrometer screw with $10 \mu \mathrm{m}$ resolution. But the specimens were cut by hand and, together with the non-constant coating thickness, the selected variation of $\pm 0.1 \mathrm{~mm}$ might be a realistic guess. All other input parameters (temperature differences and times) are the result of different averaging processes within the data processing and the residual systematical errors are guessed to be very low.

\subsection{Discussion of Thermographic Results in Transmission Configuration}

Since the investigated concrete samples are no standard materials, the real diffusivities and effusivities are unknown. Thus, the results of the different methods can only be compared but not assessed. But the limitations of the Parker method in this case are clear. The presence of enhanced heat losses $>20 \mathrm{~W} /\left(\mathrm{K} \mathrm{m}^{2}\right)$ instead of a usual value between 5 and $10 \mathrm{~W} /\left(\mathrm{K} \mathrm{m}^{2}\right)[35,36]$ causes an earlier appearance of the temperature maximum indicating a shorter penetration time for the heat, eventual leading to an enhanced diffusivity value. This systematical error could be avoided by applying the quadrupole method for evaluating the temperature transients which considers heat losses explicitly by a separate term.

The agreement between TPS and Quadrupole results is good within the estimated uncertainties and provides that the Quadrupole method in transmission configuration is suited to determine the diffusivity as well as the TPS method. 


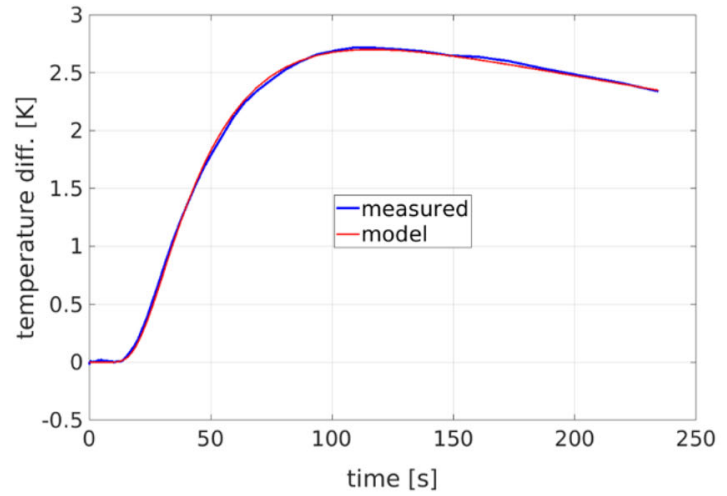

a

Fig. 8 a Comparison of experiment and model for the sample type 1 (coated) with fixed $\mathrm{h}=22 \mathrm{~W} /\left(\mathrm{m}^{2} \mathrm{~K}\right)$ and $\alpha=88,000 / \mathrm{m}$, the obtained diffusivity value is $0.77 \mathrm{~mm}^{2} / \mathrm{s}, \mathrm{qp}=0.0004$. $\mathbf{b}$ comparison of experi-

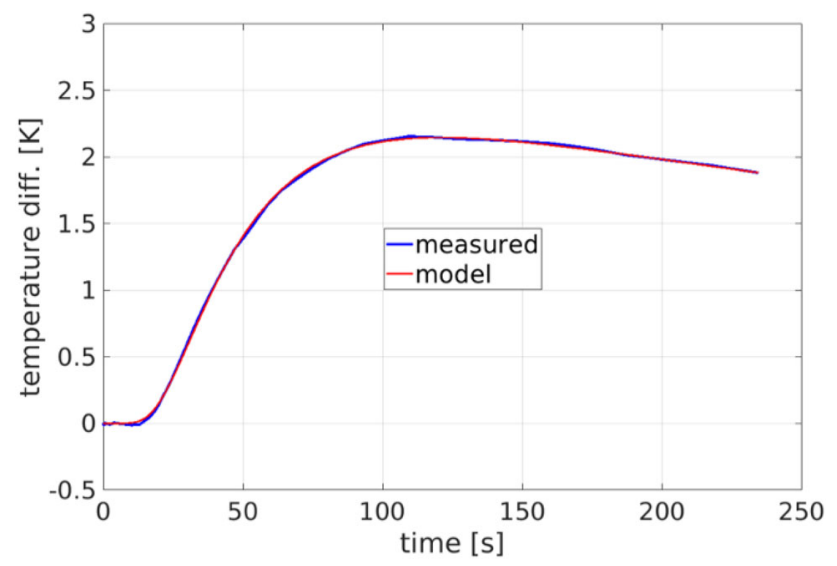

Fig. 9 Comparison of experiment and model for the sample type 1 (uncoated) with $\mathrm{h}\left(21.7 \mathrm{~W} /\left(\mathrm{m}^{2} \mathrm{~K}\right)\right)$ and $\alpha(890 / \mathrm{m})$ as fit parameters, the obtained diffusivity value is $0.71 \mathrm{~mm}^{2} / \mathrm{s}, \mathrm{qp}=0.00012$

\section{Measurement in Reflection Geometry}

While the transmission geometry requires a well-defined thickness in the range of few centimeters and access to both sides of the structure, a measurement in reflection geometry is performed on only one surface of the object with at least some centimeters thickness. This is exactly the scenario on a construction site or on an existing building. As described by Eq. 5, the effusivity of the material can be deduced in this case from the temperature decrease after a short heating pulse, when the absorbed energy is known. Here, we conducted measurements in the reflection geometry under laboratory conditions at the same specimen to enable a real comparison of both configurations.

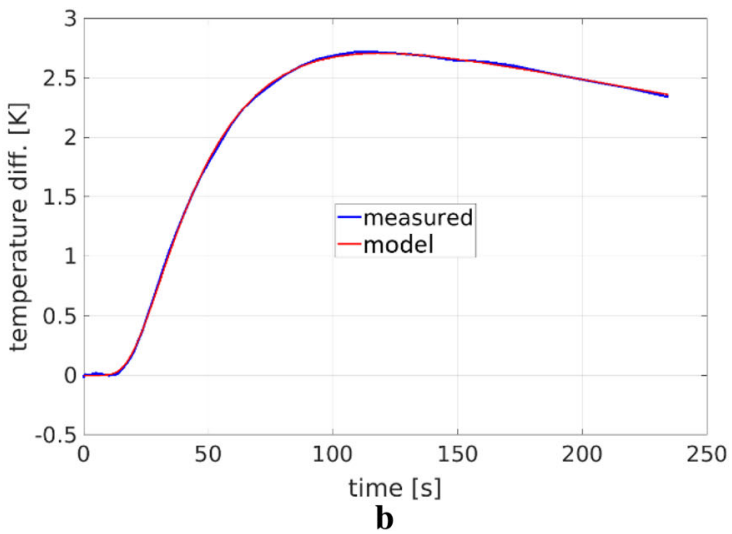

ment and model for the sample type 1 (coated) with h (obtained value $=24 \mathrm{~W} /\left(\mathrm{m}^{2} \mathrm{~K}\right)$ ) and $\alpha$ (obtained value $=890 / \mathrm{m}$ ) as fit parameters, the obtained diffusivity value is $0.72 \mathrm{~mm}^{2} / \mathrm{s}, \mathrm{qp}=0.00013$

\subsection{Measurement Setup in Reflection Geometry}

Figure 10 shows the measurement setup in reflection geometry under laboratory conditions, where the sample side heated by the laser beam is being observed by the IR-camera. The experimental equipment was the same as in case of the measurements in transmission geometry (see above), except for the following parameters:

- Heating pulse length: $0.5 \mathrm{~s}$

- Distance between camera and sample surface: $64 \mathrm{~cm}$

- Resulting spatial resolution: 5 pixels/mm

As "lessons learned" the contact area between samples and ground were drastically reduced to suppress heat losses into the ground.

\subsection{Results of Measurements in Reflection Geometry}

Figure 11 shows a photo and a thermogram of the type 1 sample (cut surface) revealing different grains within the cement matrix. The thermogram on the left side demonstrate grain-related thermal contrasts in both parts, uncoated as well as coated. Considering the uncoated part in the upper right quarter, one can easily observe the correlation between darker grains in the photo and hots spots in the thermogram. Since the grains are a component of the concrete, the measurement should include all components according to their volume share. Thus, the regions used for the transient evaluation have to be representative for the material and must not be too small. Both regions, in the coated (black rectangle) and in the uncoated (white rectangle) part, are indicated in Fig. 11a. 
Fig. 10 a Measurement setup in reflection geometry: a laser as heat source (lower right corner) illuminates a square onto the specimen type 2; IR-camera (lower left edge) with indicated optical axis (green arrow). b Illuminated area (positioning laser) in case of specimen type 1 (exposure time for the photo $0.5 \mathrm{~s})$

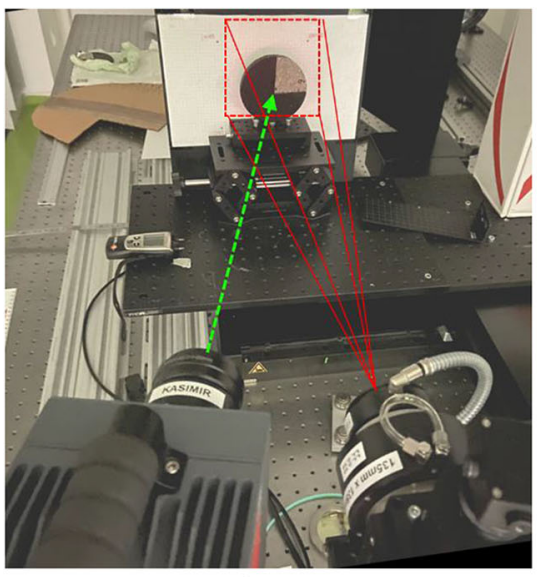

a

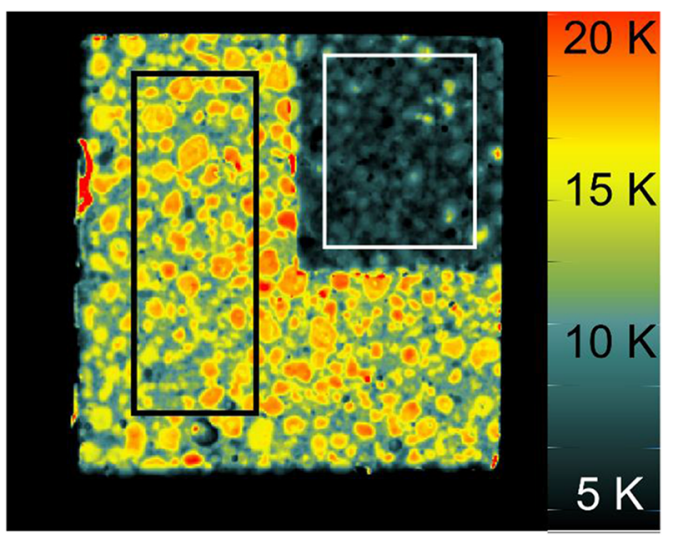

$\mathbf{a}$

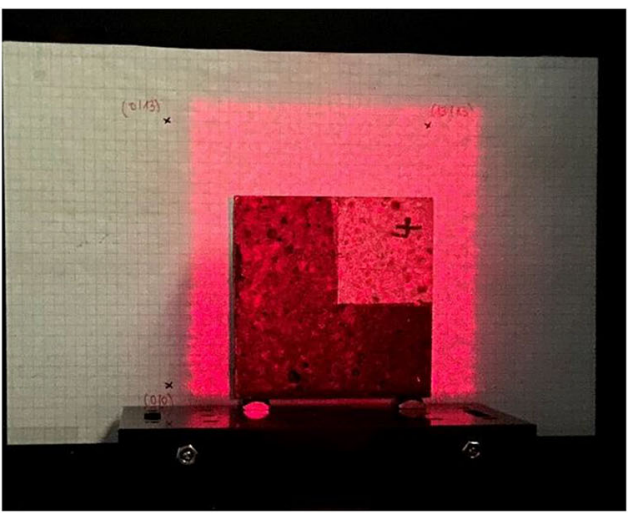

b

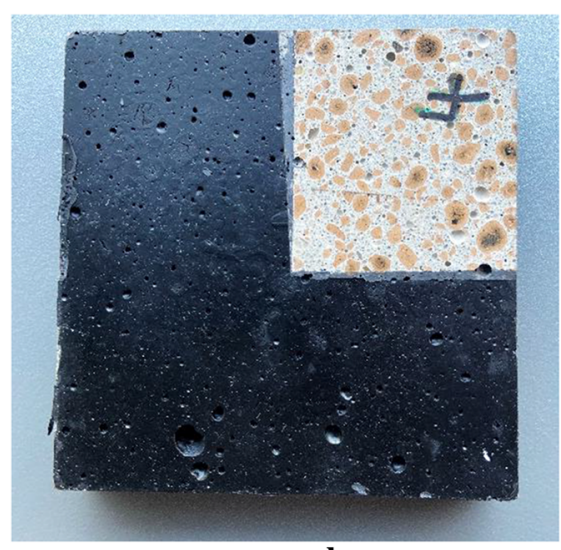

b
Interestingly, a grainy structure is visible in the coated part, too. This can be explained by different thermal properties of the grains covered by the coating.

However, Fig. 12 exhibits two further thermograms recorded during the cooling period. The hot spots were blurred but remained within the coated part after $3 \mathrm{~s}$ (Fig. 12a) but disappeared completely after $30 \mathrm{~s}$ (Fig. 12b). The remaining blotchy structure in the uncoated part of the thermogram with remaining sharp grains boundaries appeared in the transmission measurements (seen Fig. 6c) too. They are associated with low emissivity contrasts at the shear concrete surface and will not be considered here due to their low influence on the following data evaluations.

Figure 13a shows the temperature transients obtained at the selected surface regions (as indicated in Fig. 11a by different rectangles). The temperature transient of the coated part reached a higher maximum, not surprising sincethe coating absorbs more energy than the uncoated region. The comparison with the blue line provides the validity of the simple model described by Eq. 5 . In order to complete the comparison, the quadrupole method was also applied to the transients and the result is shown in Fig. 13b for the uncoated region. It is not surprising that this model with more available parameters is also well suited to describe the experimental data. Two details of the fit are remarkable: the high value of the absorption coefficient and the negative value for the thermal heat loss parameter $\mathrm{h}$. The obtained absorption coefficient is larger by factor 8.6 than in case of the transmission measurement, although surface and material are the same. It is out of the scope of this contribution to discuss this difference in detail because it would require a sensitivity analysis of the different fitting parameters in relation to the respective observation mode (transmission or reflection). The negative value of the heat loss parameter $h$ can be associated with a lateral heat flow from the coated parts leading to a weak heat gain. Please note, that the fit quality is excellent regarding the temporal range of the data set which was much longer than in case of the transmission measurements.

Finally, the obtained effusivity values were compiled in Table 4. The uncertainties of the thermographic results were estimated using the standard deviation of temperature values within the selected regions and regarding the uncertainty of 
Fig. 12 a Difference thermogram of the type 1 sample, recorded $3 \mathrm{~s}$ after the heating period. $\mathbf{b}$ difference thermogram of the type 1 sample, recorded $30 \mathrm{~s}$ after the heating period
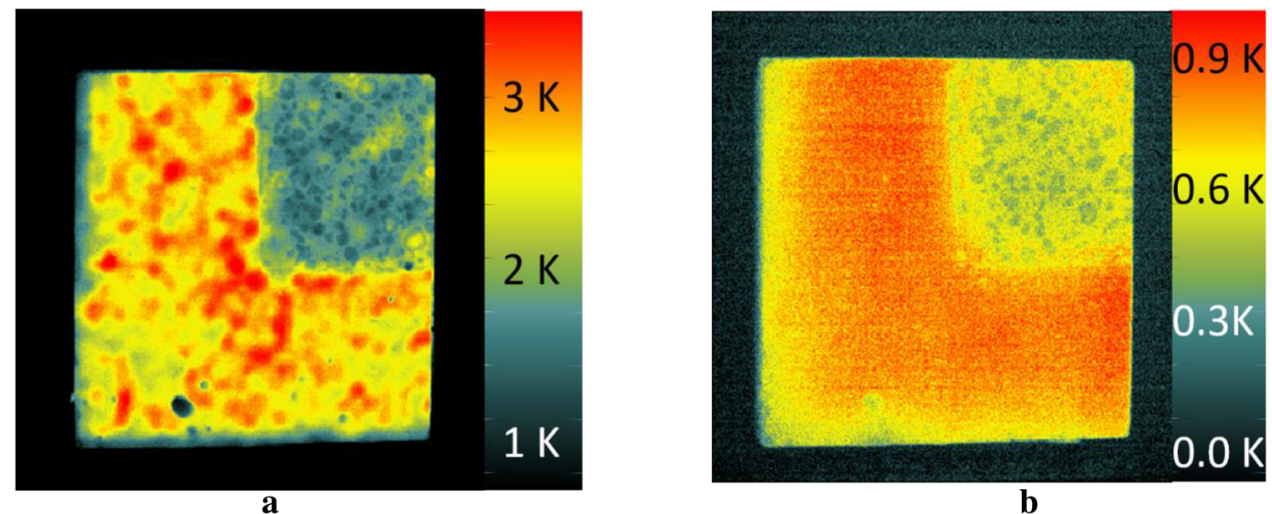

b

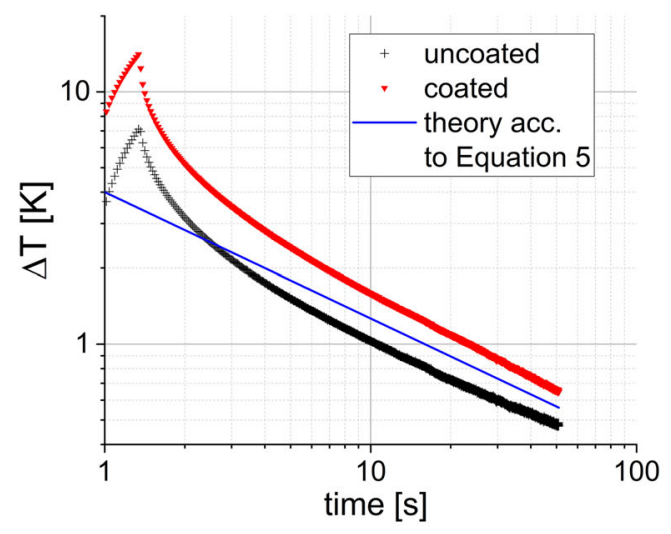

a

Fig. 13 a Difference temperature transients measured in the coated and the uncoated part of the sample type 1, the blue line indicates a transient according to Eq. 5. b Fit of the temperature transient in the uncoated

Table 4 Effusivities measured with active thermography and with TPS method (in $\mathrm{J} /\left(\mathrm{Km}^{2} \sqrt{ } \mathrm{s}\right)$ ) for the investigated specimens

\begin{tabular}{|c|c|c|c|}
\hline & Sample Type 1 & Sample Type 2 & Sample Type 3 \\
\hline $\begin{array}{l}\text { Accord. to Eq. } 5 \\
\text { uncoated }\end{array}$ & $\begin{array}{l}1550 \\
(-360+300)\end{array}$ & $\begin{array}{l}2050 \\
(-260+290)\end{array}$ & $\begin{array}{l}1480 \\
(-220+260)\end{array}$ \\
\hline $\begin{array}{l}\text { Accord. to Eq. } 5 \\
\text { coated }\end{array}$ & $\begin{array}{l}1570 \\
(-200+210)\end{array}$ & $2180 \pm 260$ & $\begin{array}{l}1550 \\
(-200+230)\end{array}$ \\
\hline $\begin{array}{l}\text { Quadrupole method } \\
\text { uncoated }\end{array}$ & 1630 & & \\
\hline TPS coated & $1690 \pm 130$ & $2180 \pm 130$ & $1835 \pm 350$ \\
\hline
\end{tabular}

the power density. This is clearly an overestimation because it based on only one data point at a certain time. Regarding instead the entire linear part of the transient curve the resulting temperature uncertainty disappears and only the uncertainty of the power density determines the entire uncertainty. It will be reduced to about \pm 150 for type 2 and even to \pm 100 for type 1 .

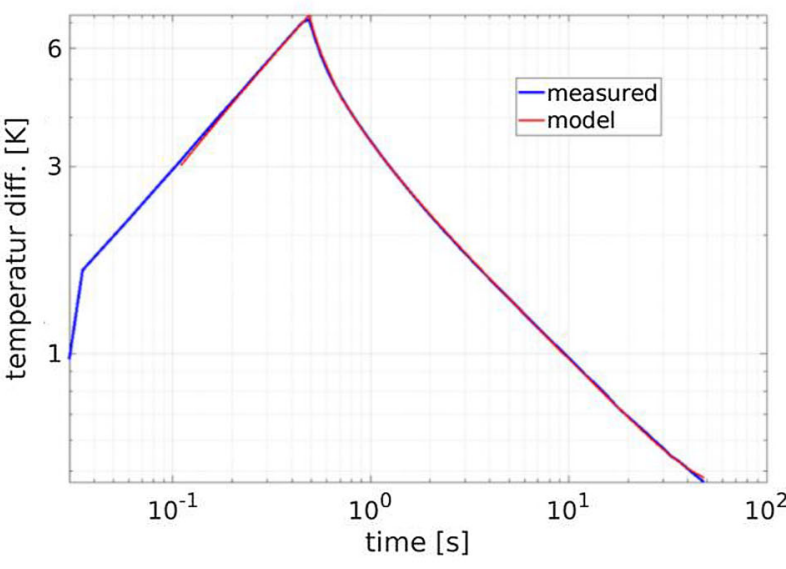

b

region without hot spots by means of the quadrupole method with $\alpha$ $(7750 / \mathrm{m}), \mathrm{h}\left(-5.7 \mathrm{~W} /\left(\mathrm{K} \mathrm{m}^{2}\right)\right)$ and the effusivity $\left(1630 \mathrm{~W} \mathrm{~s}^{0.5} / \mathrm{K} / \mathrm{m}^{2}\right)$ as fitting parameters, $\mathrm{qp}=0.0004$

\subsection{Discussion of the Thermographic Results in Reflection Configuration}

The comparison with the results of the TPS method shows a very good agreement for sample type 1 and sample type 2. In case of sample type 3, larger deviations occurred, but the uncertainty of the TPS method was remarkably increased, perhaps due to some contact issues between coating and concrete. Considering the uncertainties of both methods, a region of overlapping values exists, however.

This means that both methods allow to measure the thermal effusivity with similar precision. However, the occurred measurement uncertainties have various causes: In thermography, the main measurement uncertainty was caused by the absorptivity variance across the surface. In the TPS method, the measurement uncertainty was caused by the fact that the material concrete, which is inhomogeneous on a small scale, is measured at a series of different surface points. Thus, the variance of the values represents this thermal inhomogeneity, while the variance of the thermographic results is mainly 
caused by the optically inhomogeneous concrete surface. The inhomogeneous composition of the concrete plays only a minor role here, because averaged transients from larger regions are regarded.

\section{Conclusions}

In this study, we measured the thermal properties of different kinds of concrete with active thermography using laser irradiation as heat source with the simplest form of heating scheme: a constant heating phase with no modulation in time. Using cut samples with thicknesses between 1 and $2 \mathrm{~cm}$ the measurements could be performed in both configurations: in transmission as well as in reflection. The obtained results were compared with those from the TPS method as a standard method with a commercially available device. In case of the investigated 3 different concrete samples a good agreement was obtained between thermographic and TPS method without additional coating on the concrete.

The evaluation of transmission measurements requires a more detailed model for calculations including thermal losses and additionally a semitransparency parameter. The simple Parker formula turns out to be not sufficient under the given experimental conditions. In contrast, the measurements in reflection configuration could be evaluated by a very simple analytical solution (Eq. 5), but its application requires an additional measurement value: the absorbed heating power or energy. In this study, the required value was estimated by using a well-defined laser spot as heating source and a separate determination of the reflection coefficient at the laser wavelength. The obtained results in reflection configuration open a way to realize a mobile device suited for onsite applications. Considering the observed temperature transients with the applied energy densities, a microbolometer camera might be sufficient, but the optical properties of the concrete surface must be known in the related wavelength range. Due to the large power density of the laser safety issues must be regarded, an optical shielding will be necessary for a mobile device.

Having a value of the effusivity would allow to assess the quality of the concrete or (after a calibration) to estimate the actual moisture content or the porosity as reported earlier. However, the penetration depth of this method is restricted to the first centimeters below the surface.

Further research work is scheduled to investigate more kinds of concrete as well as the influence of moisture and fatigue state on the obtained results. Finally, further applications in other fields with only-side access are possible but depend always on the specific optical properties of the surface to be investigated.
Acknowledgements The concrete samples were provided by our colleague Götz Hüsken from division 7.2 at the BAM. Special thanks are due to our colleagues Simon Altenburg and Raphael Bernegger for their permanent support in handling the codes for the Quadrupole method.

Funding Open Access funding enabled and organized by Projekt DEAL. The research work was initiated and partially financed in the frame of the WIPANO-project 03TNG011A, funded by the Federal Ministry for Economic Affairs and Energy (BMWi).

Data Availability All data sets will be provided on request, but only in the existing format. Some data are only available in a proprietary format (*.irb for thermographic sequences).

Code Availability Not applicable.

\section{Declarations}

Conflict of interest The authors Rainer Krankenhagen and Florian Jonietz declare the following personal relationships which may be considered as potential competing interests: both are named as inventors in a related patent application but are not involved in financial matters. The author Stefan Zirker has no known competing financial interests or personal relationships that could have appeared to influence the work reported in this paper.

Open Access This article is licensed under a Creative Commons Attribution 4.0 International License, which permits use, sharing, adaptation, distribution and reproduction in any medium or format, as long as you give appropriate credit to the original author(s) and the source, provide a link to the Creative Commons licence, and indicate if changes were made. The images or other third party material in this article are included in the article's Creative Commons licence, unless indicated otherwise in a credit line to the material. If material is not included in the article's Creative Commons licence and your intended use is not permitted by statutory regulation or exceeds the permitted use, you will need to obtain permission directly from the copyright holder. To view a copy of this licence, visit http://creativecomm ons.org/licenses/by/4.0/.

\section{References}

1. Chaffar, K., Chauchois, A., Defer, D., Zalewski, L.: Thermal characterization of homogeneous walls using inverse method. Energy Build. 78, 248-255 (2014). https://doi.org/10.1016/j.enbuild.2014. 04.038

2. Sassine, E., Cherif, Y., Antczak, E.: Parametric identification of thermophysical properties in masonry walls of buildings. J. Build Eng. (2019). https://doi.org/10.1016/j.jobe.2019.100801

3. Yang, Y.Y., Wu, T.V., Sempey, A., Dumoulin, J., Batsale, J.C.: Short time non-destructive evaluation of thermal performances of building walls by studying transient heat transfer. Energy Build. 184, 141-151 (2019). https://doi.org/10.1016/j.enbuild.2018.12.002

4. Altenburg, S.J., Krankenhagen, R., Bavendiek, F.: Thickness determination of polymeric multilayer surface protection systems for concrete by means of pulse thermography. AIP Conf. Proc. 1806(1), 100004 (2017). https://doi.org/10.1063/1.4974669

5. Wang, H.J., Hsieh, S.J.: Solving the inverse heat conduction problem in using long square pulse thermography to estimate coating thickness by using SVR models based on restored pseudo heat flux 
(RPHF) in-plane profile. J. Nondestruct. Eval. 37(4), 2018 (2018). https://doi.org/10.1007/s10921-018-0535-8

6. Cobirzan, N., Balog, A.A., Belean, B., Borodi, G., Dadarlat, D., Streza, M.: Thermophysical properties of masonry units: accurate characterization by means of photothermal techniques and relationship to porosity and mineral composition. Constr Build Mater 105, 297-306 (2016). https://doi.org/10.1016/j.conbuildmat.2015. 12.056

7. Plasser, H., Mayr, G., Thummerer, G., Hendorfer, G., Burgholzer, P., Major, Z.: Photothermal porosity estimation in CFRP by the time-of-flight of virtual waves. J Nondestruct Eval (2020). https:// doi.org/10.1007/s10921-020-00722-0

8. Zgueb, R., Brichni, A., Yacoubi, N.: Improvement of the thermal properties of Sorel cements by polyvinyl acetate: consequences on physical and mechanical properties. Energy Build. 169, 1-8 (2018). https://doi.org/10.1016/j.enbuild.2018.03.007

9. Zhao, D.L., Qian, X., Gu, X.K., Jajja, S.A., Yang, R.G.: Measurement techniques for thermal conductivity and interfacial thermal conductance of bulk and thin film materials. J. Electron. Packag. 138(4), 2016 (2016). https://doi.org/10.1115/1.4034605

10. Gustavsson, M., Karawacki, E., Gustafsson, S.E.: Thermalconductivity, thermal-diffusivity, and specific-heat of thin samples from transient measurements with hot disk sensors. Rev Sci Instrum 65(12), 3856-3859 (1994). https://doi.org/10.1063/1.1145178

11. He, Y.: Rapid thermal conductivity measurement with a hot disk sensor: Part 1. Theoretical considerations. Thermochim. Acta 436(1), 122-129 (2005). https://doi.org/10.1016/j.tca.2005.06.026

12. ISO 22007-2:2015 - Plastics - Determination of thermal conductivity and thermal diffusivity - Part 2: Transient plane heat source (hot disc) method, 2015

13. Kumar, V., Dixit, U.S., Zhang, J.: Determination of thermal conductivity, absorptivity and heat transfer coefficient during laser-based manufacturing. Measurement 131, 319-328 (2019). https://doi. org/10.1016/j.measurement.2018.08.072

14. Ukrainczyk, N., Matusinović, T.: Thermal properties of hydrating calcium aluminate cement pastes. Cem. Concr. Res. 40(1), 128-136 (2009). https://doi.org/10.1016/j.cemconres.2009.09.005

15. Breuer, S., Schwotzer, M., Speziale, S., Schilling, F.R.: Thermoelastic properties of synthetic single crystal portlandite $\mathrm{Ca}(\mathrm{OH})_{2}$ - Temperature-dependent thermal diffusivity with derived thermal conductivity and elastic constants at ambient conditions. Cem. Concr. Res. 137, 106199 (2020). https://doi.org/10.1016/j. cemconres.2020.106199

16. ASTM C714-05, Standard Test Method for Thermal Diffusivity of Carbon and Graphite by Thermal Pulse Method, 2005

17. ASTM E 1461 - 13, Standard Test Method for Thermal Diffusivity by the Flash Method

18. Liu, K., Wang, Z., Jin, C., Wang, F., Lu, X.Y.: An experimental study on thermal conductivity of iron ore sand cement mortar. Constr Build Mater 101, 932-941 (2015). https://doi.org/10.1016/ j.conbuildmat.2015.10.108

19. Santhosh, U., Gowayed, Y., Ojard, G., Smyth, I., Kalarikkal, S., Jefferson, G.: Quantification of porosity in ceramic matrix composites using thermography. J Nondestruct Eval 37(2), 2018 (2018). https://doi.org/10.1007/s10921-018-0487-z

20. Soares, N., Martins, C., Goncalves, M., Santos, P., da Silva, L.S., Costa, J.J.: Laboratory and in-situ non-destructive methods to evaluate the thermal transmittance and behavior of walls, windows, and construction elements with innovative materials: a review. Energy Build. 182, 88-110 (2019). https://doi.org/10.1016/j.enbuild.2018. 10.021
21. Parker, W.J., Jenkins, R.J., Abbott, G.L., Butler, C.P.: Flash method of determining thermal diffusivity, heat capacity, and thermal conductivity. J. Appl. Phys. 32(9), 1679 (1961). https://doi.org/10. 1063/1.1728417

22. H. D. AB. Homepage: Hot Disk AB division instruments. https:// www.hotdiskinstruments.com/products-services/instruments/tps$1500 /$

23. H. D. AB. Hot Disk Thermal Constants Analyser Manual, Hot Disk AB, Gothenburg, Sweden, 2019.

24. DIN 4108-4: Thermal insulation and energy economy in buildings - Part 4: Hygrothermal design values, 2013

25. ASTM E1933-14(2018), Standard Practice for Measuring and Compensating for Emissivity Using Infrared Imaging Radiometers, A. International, 2018

26. Muller, J.P., Krankenhagen, R.: Optimizing thermographic testing of thick GFRP plates by assessing the real energy absorbed within the material. Compos Struct 215, 60-68 (2019)

27. Krankenhagen, R., Worzewski, T., Maierhofer, C.: Cooling-down of thermal thick probes after flash excitation - a measure for the real energy density? Infrared Phys. Technol. 72, 258-265 (2015). https://doi.org/10.1016/j.infrared.2015.07.014

28. Hahn, D.W., Özisik, M.N.: Heat Conduction, 3rd edn. Wiley, Hoboken (2012)

29. Almond, D.P., Patel, P.M.: Photothermal Science and Techniques (PHYSICS AND ITS APPLICATION), p. 241. Chapman \& Hall, London (1996)

30. DIN EN 821-2: Advanced technical ceramics - Monolithic ceramics - Thermo-physical properties - Part 2: Determination of thermal diffusivity by the laser flash (or heat pulse) method, 1997.

31. ISO 18755, Fine ceramics (advanced ceramics, advanced technical ceramics) - Determination of thermal diffusivity of monolithic ceramics by laser flash method, 2005

32. ISO/DIS 13826, Metallic and other inorganic coatings - Determination of thermal conductivity of thermally sprayed ceramic coatings by laser flash method, 2011

33. ISO 22007-4:2008 - Plastics - Determination of thermal conductivity and thermal diffusivity - Part 4: Laser flash method, 2008

34. Maillet, D., Andre, S., Batsale, J.C., Degiovanni, A., Moyne, C.: Thermal Quadrupoles: Solving the Heat Equation through Integral Transforms. Wiley, New York (2000)

35. Salazar, A., Mendioroz, A., Apinaniz, E., Pradere, C., Noel, F., Batsale, J.C.: Extending the flash method to measure the thermal diffusivity of semitransparent solids. Meas Sci Technol 25(3), 2014 (2014). https://doi.org/10.1088/0957-0233/25/3/035604

36. Altenburg, S.J., Weber, H., Krankenhagen, R.: Thickness determination of semitransparent solids using flash thermography and an analytical model. Quant. InfraRed Thermogr. J. 15(1), 95-105 (2017). https://doi.org/10.1080/17686733.2017.1331655

Publisher's Note Springer Nature remains neutral with regard to jurisdictional claims in published maps and institutional affiliations. 